\title{
Integration Concept of the Reflectometry Diagnostic for the Main Plasma in DEMO
}

\begin{tabular}{|c|c|}
\hline Journal: & IEEE Transactions on Plasma Science \\
\hline Manuscript ID & Draft \\
\hline Manuscript Type: & Special Issue - Selected Papers from SOFE 2017 \\
\hline Date Submitted by the Author: & $\mathrm{n} / \mathrm{a}$ \\
\hline Complete List of Authors: & $\begin{array}{l}\text { Malaquias, Artur; Instituto de Plasmas e Fusao Nuclear, physics } \\
\text { Silva, Antonio; Instituto de Plasmas e Fusao Nuclear } \\
\text { Moutinho, Ruben; Instituto de Plasmas e Fusao Nuclear } \\
\text { Luis, Raul; Instituto de Plasmas e Fusao Nuclear, physics } \\
\text { Lopes, Andre; Instituto de Plasmas e Fusao Nuclear, physics } \\
\text { Quental, Paulo; Instituto de Plasmas e Fusao Nuclear, physics } \\
\text { Prior, Luis; Instituto de Plasmas e Fusao Nuclear, physics } \\
\text { Velez, Nicolau; Instituto de Plasmas e Fusao Nuclear, physics } \\
\text { Policarpo, Hugo; Instituto de Plasmas e Fusao Nuclear, physics } \\
\text { Vale, Alberto; Instituto de Plasmas e Fusao Nuclear, physics } \\
\text { Biel, Wolfgang; Forschungszentrum Juelich, IEK-4 Plasma Physics Juelich, } \\
\text { NRW, Germany, IEK-4 Plasma Physics; Universiteit Gent, Department of } \\
\text { Applied Physics } \\
\text { Aubert, Julien; CEA Direction des Sciences de la Matiere } \\
\text { Reungoat, Mathieu; Centrum výzkumu Řež } \\
\text { Cismondi, Fabio; EUROFUSION Consortium, PPPT } \\
\text { Franke, Thomas; EUROFUSION Consortium, PPPT; Max-Planck-Institut fur } \\
\text { Plasmaphysik }\end{array}$ \\
\hline Key Words: & $\begin{array}{l}\text { Reflectometry, Fusion reactors, Tokamaks, Plasma control, Density } \\
\text { measurement, Magnetic confinement }\end{array}$ \\
\hline Specialty/Area of Expertise: & Fusion Plasmas Diagnostics \\
\hline
\end{tabular}

\section{SCHOLARONE ${ }^{\text {m }}$}




\title{
Integration Concept of the Reflectometry Diagnostic for the Main Plasma in DEMO
}

\author{
A. Malaquias, A. Silva, R. Moutinho, R. Luis, A. Lopes, P. B. Quental, L. Prior, N. Velez, H. \\ Policarpo, A. Vale, W. Biel, J. Aubert, M. Reungoat, F. Cismondi and T. Franke
}

\begin{abstract}
Abstrat-This paper presents the first case study of integration of reflectometry antennas and waveguides located at several poloidal angular positions covering a full poloidal section of the Helium Cooled Lithium Lead breading blanket. The integration shall satisfy strong machine driven constraints (in addition to the physics performance). Diagnostic components installed in the blanket segments must: i) survive for the all period between blanket replacement, ii) be remote handling (RH) compatible with blanket, iii) behave thermomechanical as the blanket structure, iv) cross with integrity the vacuum and reference boundaries (vessel/cryostat/building) and tolerate their relative displacements and $v$ ) be compatible with the blanket shielding and cooling services. The present solution developed so far respects several of the main constraints namely, RH compatibility with the full blanket segment and its thermomechanical properties and cooling compatibility but also identifies important issues on the interfaces between the diagnostic antennae extensions and the pipe services at the vessel and also interfaces between vessel and cryostat requiring challenging RH and self-alignment solutions to be demonstrated. Monte Carlo neutronic simulations have been done in order to evaluate the heat loads and shielding capabilities of the system. The first results indicate that the cooling for the EUROFER diagnostic components (antennas and waveguides) can in principle be provided by the blanket cooling services (He is considered) via connection to the main Back Supporting Structure (BSS) and routed via the main diagnostic structure body to specific hot spots in the antennas.
\end{abstract}

Index Terms - Reflectometry, Fusion reactor design, Tokamaks, Plasma diagnostics, Plasma density, Magnetic confinement

Manuscript submitted on $8^{\text {th }}$ June 2016 . This work has been carried out within the framework of the EUROfusion Consortium and has received funding from the Euratom research and training programme 2014-2018 under grant agreement No 633053. The reviews and opinions expressed herein do not necessarily reflect those of the European Commission.

A. Malaquias, A. Silva, R. Moutinho, R. Luis, A. Lopes, P. B. Quental, L. Prior, N. Velez, H. Policarpo, A. Vale, are with the Instituto dos Plasmas e Fusão Nuclear, Instituto Superior Técnico, Universidade de Lisboa, 1049-001 Lisboa, Portugal (e-mail: artur.malaquias@ ipfn.tecnico.ulisboa.pt).

W. Biel is within the Institute of Energy and Climate Research, Forschungszentrum Jülich GmbH, Germany and the Department of Applied Physics, Ghent University, Belgium.

J. Aubert is with the CEA- Saclay, DEN, DM2S, F-91191 Gif-sur-Yvette, France.

M. Reungoat is with the Centrum výzkumu Řež, Hlavní 130, 25068 Husinec - ̌̌žz, Czech Republic

F. Cismondi is with the EUROfusion Consortium - PPPT department, Boltzmannstrasse 2, 85748 Garching, Germany

T. Franke is with the EUROfusion Consortium - PPPT department, Boltzmannstrasse 2, 85748 Garching, Germany and the Max-Planck-Institut für Plasmaphysik, Boltzmannstr. 2, D-85748 Garching, Germany

\section{INTRODUCTION}

$F_{d}$ ROM the control point of view DEMO fusion power plant demands a reliable diagnostic capability based on a minimal set of robust individual diagnostic systems. The overall diagnostic system must be accurate in detecting plasma disruptive conditions and exhibit robustness to the relatively large radiation flux and fluence levels. The individual diagnostic systems must provide for the required measurement accuracy, dynamic range coverage and achieve an acceptable long life time. The reflectometry diagnostic may present several advantages from the point of view of radiation robustness and components life time. From the hardware perspective it contains antennas and waveguides that can be long living under the radiation fluxes and plasma induced deposition/erosion processes expected for DEMO. The role of reflectometry diagnostic for DEMO is twofold: i) to provide the radial density profile at several poloidal angles (2D map) and ii) to provide data for the feedback control for plasma position and shape. Several groups of antennas need to be distributed along the poloidal section in a number that can satisfy the DEMO control requirements. The study of diagnostic performance and control requirements definition is being developed and several aspects regarding integration in DEMO are being assessed.

This paper describes the initial concept of reflectometry implementation in DEMO. Ray-tracing results and the consequences for the antennas locations and waveguides routing in DEMO are presented. In particular, the integration of reflectometry diagnostic components in the Helium Cooled Lithium Lead (HCLL) Breading Blanket (BB) was chosen as a case study. Nuclear heating induced by the neutron fluxes has been estimated and used to dimensioning cooling systems. The compatibility with BB operation and Remote Handling ( $\mathrm{RH})$ is also addressed.

\section{CONCEPT FOR REFLECTOMETRY MEASUREMENTS IN DEMO}

The requirements for reflectometry diagnostic have been derived from the control requirements for DEMO [1]. In particular, reflectometry shall provide the density profile in the gradient region (pedestal) with a spatial resolution of $\Delta_{\text {ped }} / 30$ (relaxed to a/20 in the core) and a time resolution of $\Delta \mathrm{T}<100$ $m s$. As for plasma position and shape the final requirements have not yet been developed but based on present experiments [2] 1-2 cm is being considered to guide the conceptual study, also with $\Delta \mathrm{T}<100 \mathrm{~ms}$. Reflectometry is also required to 
detect plasma instabilities, in particular Vertical Displacement Events (DVEs) assisting the magnetics in this task. Due to the radiation levels in DEMO the magnetic coils must be placed in relatively well shielded areas beyond the $\mathrm{BB}$ and vessel limiting the sensivity of the magnetic diagnostics. In addition, well known integration drift effects induced by electric noise will limit the capability of magnetic sensors do accurately detect VDEs. The implications for reflectometry to be used for plasma position and shape control is that in order to provide sufficient coverage it should have several measuring points at several poloidal locations.

For the DEMO plasma density $\left(n_{e}\right)$ and temperature $\left(T_{e}\right)$ ranges reflectometry may operate in O-mode $(15-115 \mathrm{GHz})$ for probing the plasma edge and pedestal ( $n_{e}$ profile) and for position and shape control. As for the core measurements they can be assessed for upper cut-off X-mode operation (140 $250 \mathrm{GHz})$. The relatively high $T_{e}$ values $(>2 \mathrm{keV})$ impose a relativistic treatment for the determination of the cut-off condition and location. In addition, $\mathrm{X}$-mode operation requires information of the total magnetic field in order to determine $n_{e}$. It is foreseen that the $T_{e}$ profiles are obtained via the Electron Cyclotron Emission (ECE) and spectroscopic diagnostics while magnetic data could be obtained from the equilibria.

A simplified ray tracing model was used in order to determine the trajectory of the reflected rays during a frequency scan in O-mode. For an emitting antenna aligned at equatorial level the reflected rays will return in the same direction as the incidence ray. For a few $\mathrm{cm}$ offset the nonnormal incidence to the cut-off layer refraction effects induce an angular spread of the reflected beam, more evident for the inner cut-off layers (increased plasma surface curvature). This is illustrated in Fig. 1 where a vertical offset of $2 \mathrm{~cm}$ of the returning beam is observed for the inner-most cut-off layer. For each particular layout arrangement the receiving antennas locations and beam shape have to be optimized in order to cover for the vertical spread of the reflected beams.For injection positions from different poloidal locations (away from the equatorial level) the beams' incidence angle and shape shall be carefully optimized taking into account the fact that the shape of the flux surfaces changes stronger than at equatorial level. The effect of VDEs in the angle of incidence/reflection is quite evident as depicted in Fig. 2. Taking the case for the most favorable location for the antennas (equator) and analyzing the return path of the reflected beams from a fixed reflection layer it is observed that a plasma vertical shift of $\pm 6 \mathrm{~cm}$ will induce a similar vertical spread of the reflected beams.

Based on these results it is expected that the implementation of emitting and receiving antennas will be dependent on poloidal location. Detailed simulations will inform on the best compromise between number of antennas and beam receiving power (and optimized beam shaping) in order to be able to

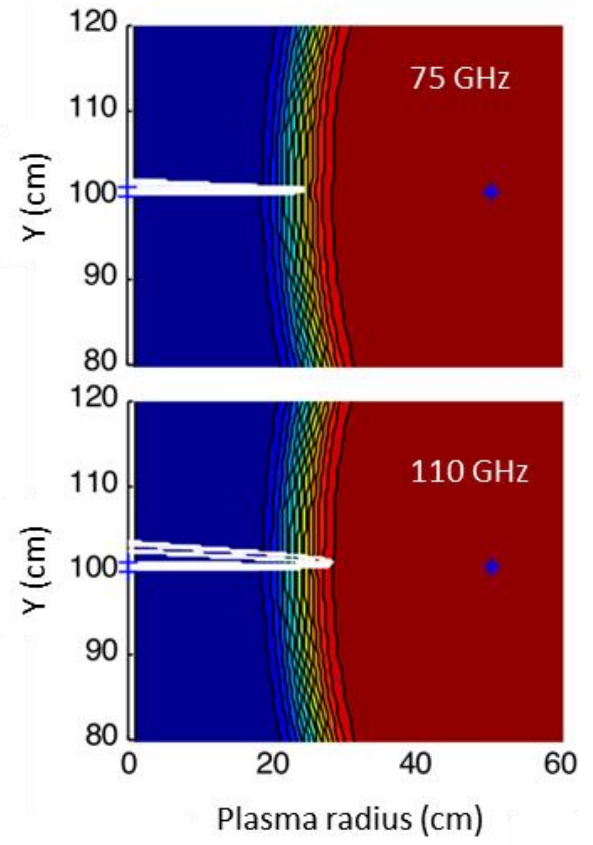

Fig. 1 - Incidence and reflected rays (O-mode) at two different radial locations. A small inclination of the incidence beam to the cut-off layer normal will induce an angular spread of the reflected rays.

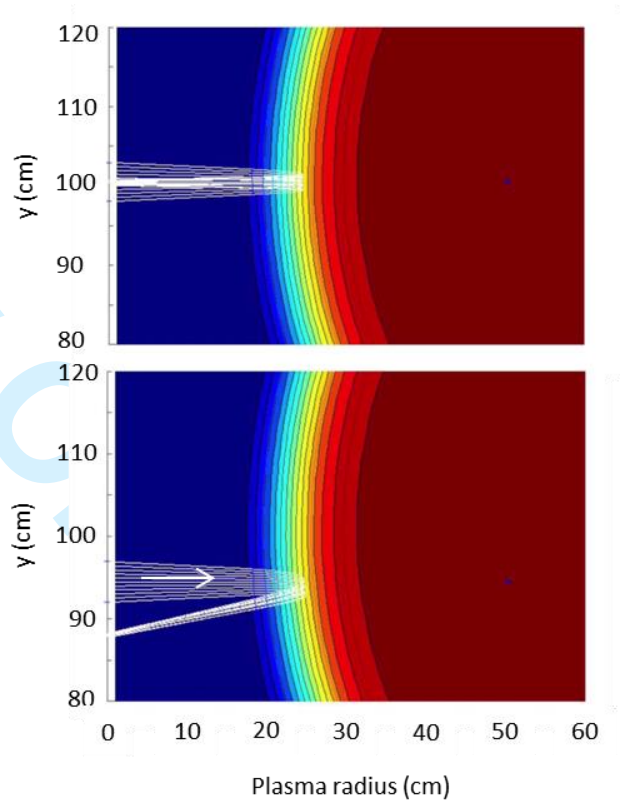

Fig. 2 - Injection at fixed frequency in two distinct vertical locations, simulating a plasma vertical displacement. The reflected beam shows a relatively large y-displacement. These must be accommodated by implementing more antennas and/or shaping the beam focus.

measure the maximum range of displacement (tens of $\mathrm{cm}$ ) without compromising the accuracy in detecting small plasma displacements (1-2 cm). In Fig. 3 it is depicted the expected qualitative performance of reflectometry as a function of poloidal location. 


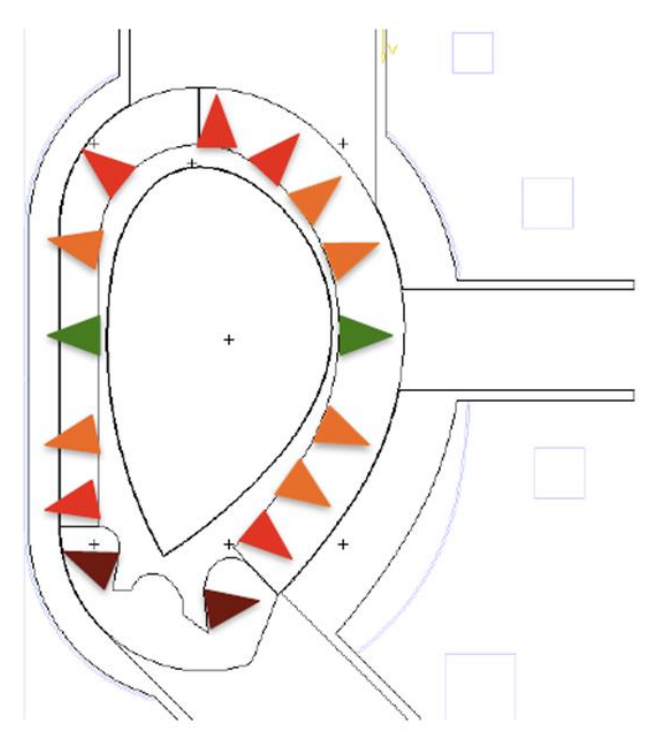

Fig. 3 - A preliminary assessment indicates that there are three main areas of different feasibility for the diagnostic: GREEN - very favorable; ORANGE performance limited in some conditions due to non-perpendicular (to flux surface) propagation of the microwaves; 3 - RED - very unlikely that the diagnostic will perform according to the requirements due to strong refraction; BROWN - divertor - not assessed.

\section{IMPLEMENTATION CONCEPT - SLIM CASSETTE}

The implementation of reflectometry requires that the antennas be placed in front of the plasma. They are considered to be made of EUROFER or have additional $\mathrm{W}$ coating. The waveguides (made of EUROFER) must be routed from the plasma front antennas up to the diagnostic hall, crossing two vacuum boundaries one, at the vessel level (primary vacuum boundary) and the other at the cryostat level (cryostat vacuum) before entering the building through the bio-shield barrier. The implementation concept is based on a construction of a dedicated dummy section of the BB modules. Several options are being discussed regarding the sharing of the first wall (FW) and the common Back Support Structure (BSS) between the BB and the dummy BB sections. The concept presented in this work is based on the independent Slim Cassette (SC) structure presented in Fig. 4 (with independent FW).

The SC independent dummy sector ( $2 x$ half-full poloidal sector) is attached to the BSS (Fig. 5). The two possibilities of attachment being considered have as common features the use of the He supply provided via the BSS and the attachment of the SC to the BSS. These requirements are to ensure that the $\mathrm{BB}$ and the $\mathrm{SC}$ are inserted / removed as one single component compatible with $\mathrm{RH}$ operations when installing and removing the BB 'banana'. One other characteristic being preserved is the segmentation between the dummy modules the same as in the BB 'banana' helping to maintain a more similar mechanical behavior.

Regardless of the $\mathrm{BB}$ integration solution to become adopted some additional integration aspects can be readily address. The remaining of the paper will describe the preliminary concepts regarding routing of waveguides, $\mathrm{RH}$ compatibility, and neutronic analysis and cooling.

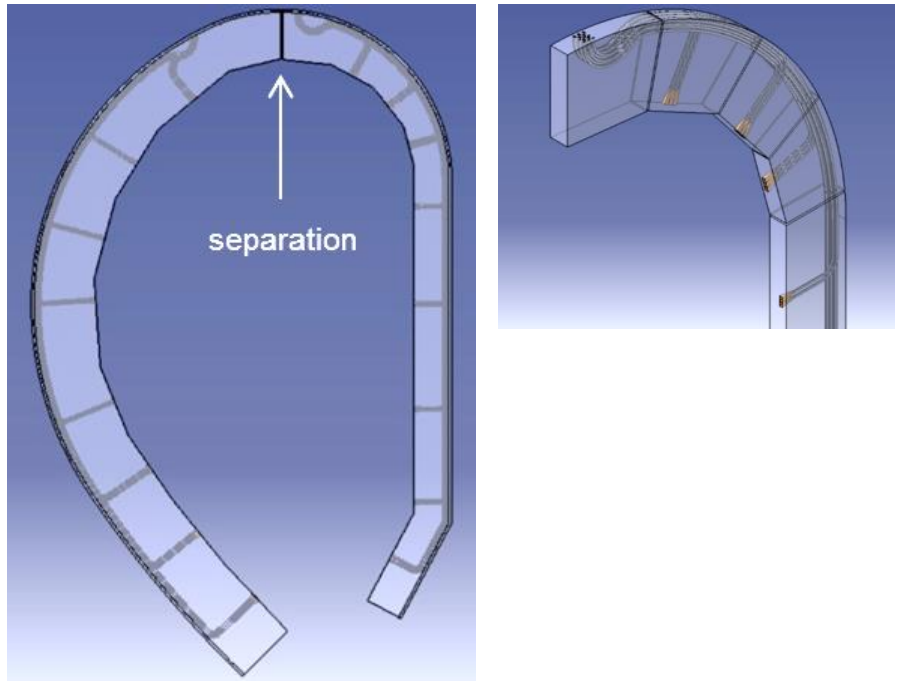

Fig. 4 - Full poloidal array of antennas and waveguides installed in an array of BB dummy modules (16 poloidal locations). The slim cassette average width is $20-25 \mathrm{~cm}$ (in toroidal direction). The dummy sectors are fixed in a common frame running at the back of the structure. The radius of curvature for the antennas is kept above $12 \mathrm{~cm}$ in order to avoid mode conversion.
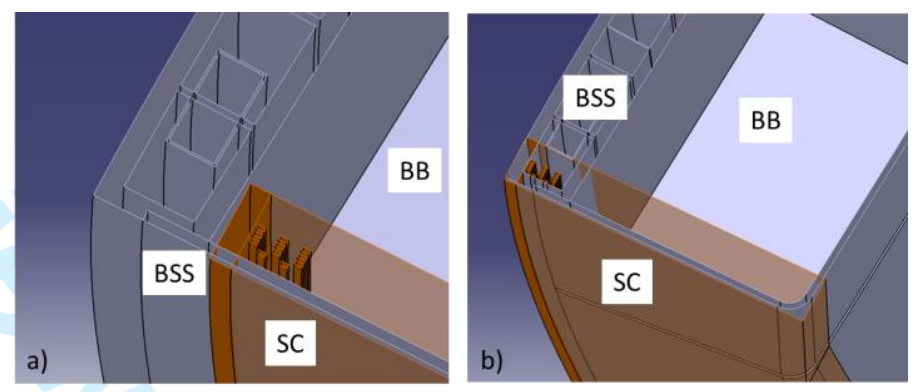

Fig. 5 - Illustration of two possible attachments: a) common BSS -the SC is fixed to the front of the BSS and b) independent SC sector - the SC is attached to the side of BSS

\section{WAVEGUIDES ROUTING AND RH COMPATIBILITY}

The SC will have interfaces with some DEMO main systems, namely, space sharing in the port, vacuum barriers, cooling system, and RH tools and procedures.

\section{A. Interfaces at the port area}

The port area is relatively crowded with service pipes for the $\mathrm{BB}$ sectors ('bananas') transporting $\mathrm{He}$ and $\mathrm{LiPb}$ from and to the BB sectors. The waveguides (WGs) installed in the SC must be routed to the building. The outboard (OB) SC sector has a more clear area for integration and routing. In this work we have investigated concepts to route the WGs from the OB $\mathrm{SC}$ sector up to the first vacuum interface i.e. vessel to cryostat. The routing is implemented via two main WGs extensions. The first bunch of waveguides extensions (the vertical extensions) have the function of converting the single mode rectangular WGs profile (inside dimensions of $19 \times 9.5$ $\left.\mathrm{mm}^{2}\right)$ into broadband circular waveguides $\left(\phi_{\text {out }}=10 \mathrm{~cm}\right)($ Fig. 6 ). The circular waveguides will provide a better option for taking the changes on direction and the accommodation of relative movements between different sections. The vertical extensions will fine align to the SC flange via the guiding 
studs. A metallic foil mask will be placed between the two flanges in order to reduce the gaps (reduce MW signal leakage). The extensions will be bolt (or fixed by other type of attachment) into the SC flange from the top using self-carrying extended bolts. Vacuum tightness is not required at this joint.
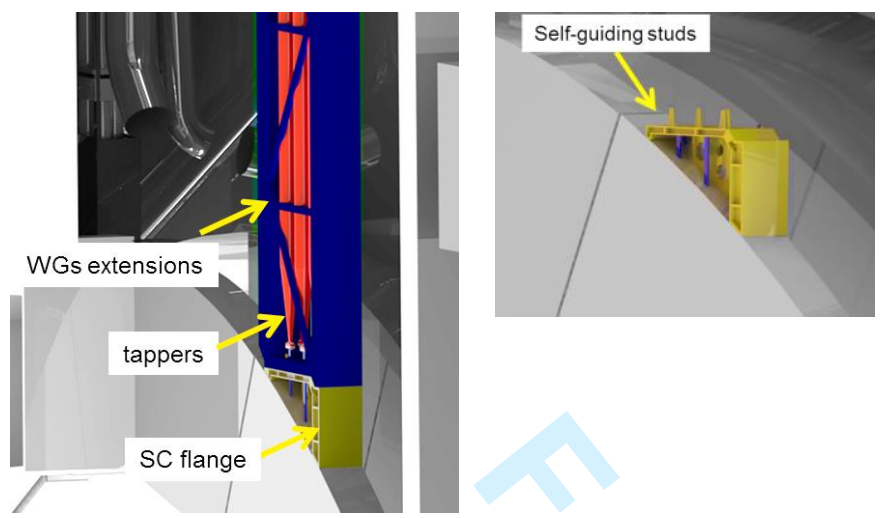

Fig. 6 - WGs extensions and SC end-connecting flange. The rectangular WGs profile is tapered to a circular profile.

The opposite end of the vertical extensions contain (single or double) miter bends and linear motion de-couplers. These are required in order to take the relative motions between the $\mathrm{BB}$ sector and the vessel port. These relative movements range from a few mm during operation to a few $\mathrm{cm}$ offset from room to operating temperature. For decoupling the relative motion and break the primary vacuum boundary a similar conceptual arrangement as in ITER 98 is proposed [3] (Fig. 7).

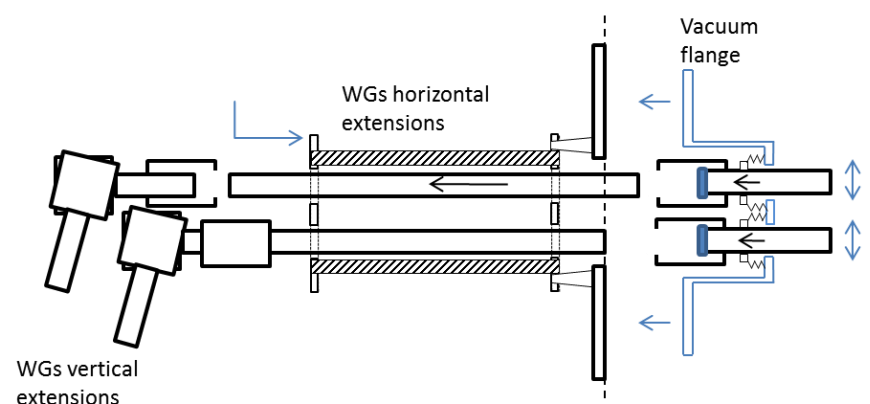

Fig. 7 - Conceptual interface between the WGs extensions and the port side flange. The horizontal WGs extension's frame is RH placed and attached to the inside of the port wall. The individual WGs are then individually coupled to the miter bends of the vertical extensions. The vacuum flange couples to the other end of the WGs and is sealed against the port wall (bolted or welded). Quartz windows in the flange WGs segments are used for establishing the primary vacuum break.

In Fig. 8 is presented the layout of the vertical and horizontal WGs extensions inside the port. The routing of the WGs in this area of the port is quite straightforward with no clashes with pipes. The interfaces are with the pipes containment barrier (Fig. 9) and with the port due to the required dedicated side opening.

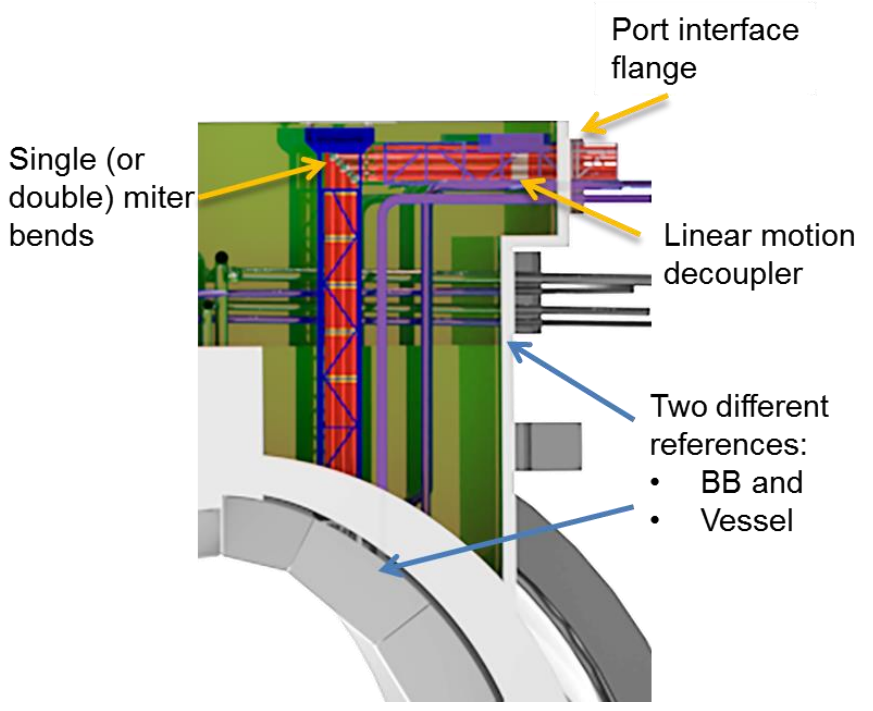

Fig. 8 - The vertical and horizontal WGs extensions coupled in place. The relative motions between $\mathrm{BB}$ and Vessel are taken by an arrangement of miter bends and linear decouplers.

The integration of the inboard WGs extensions presents some additional challenges since the space is heavily occupied with the pipe-forest of $\mathrm{He}$ and LiPb supply (Fig. 9). The IB integration has not yet been developed and will be addressed in the near future.

\section{WGs extensions} (in-board)

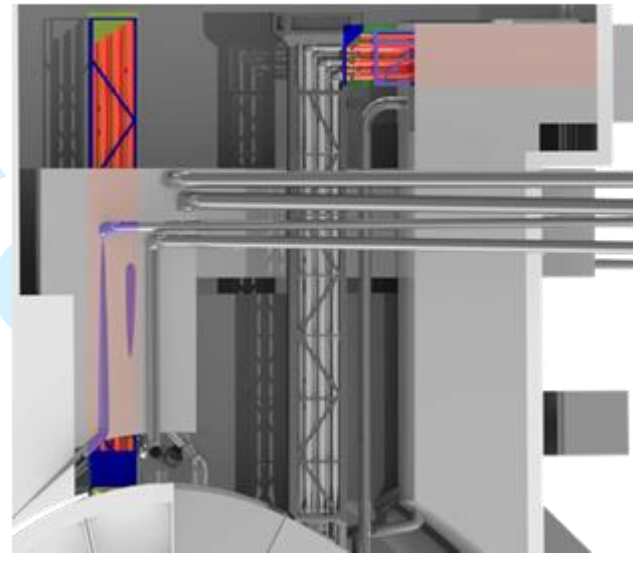

Fig. 9 - View of the interface between the IB WGs vertical extensions and the $\mathrm{BB}$ servicing pipes and its containment barrier.

\section{B. Remote handling compatibility}

The RH maintenance of the SC follows the BB sector RH procedure [4]. It is inserted/removed with the whole $\mathrm{BB}$ 'banana'. The SC is attached to the common back support structure of the BB. The He supply is also provided from the BSS. For the WGs extensions the RH procedure the standard BB transporter can be used. The WGs extension's frame contains a receiving end effector for that purpose. The movements required have been chosen in view of the blanket transporter capability and the conclusion is that the system is $\mathrm{RH}$ compatible. In Fig. $\mathbf{1 0}$ is illustrated the sequence of movements of the blanket transporter applied to the WGs vertical extensions. 

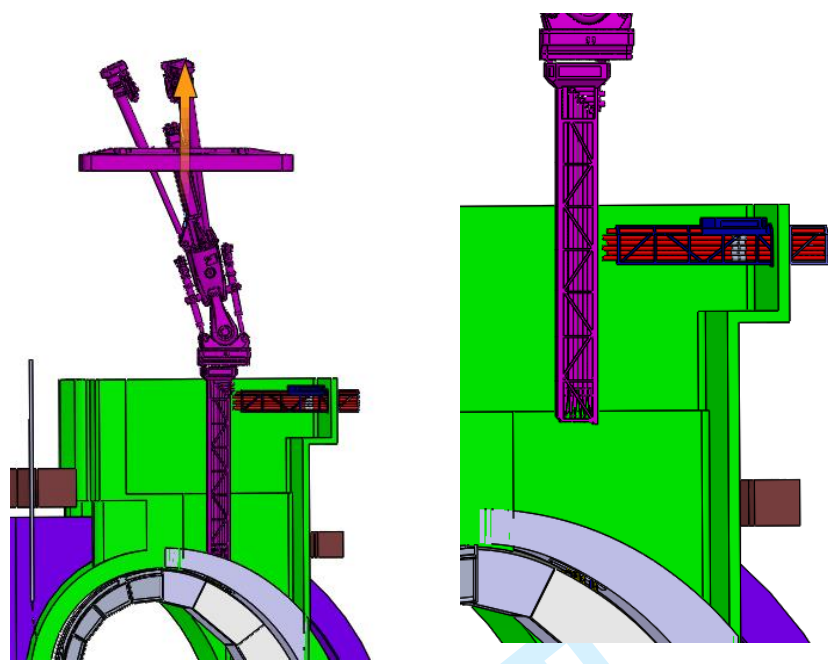

Fig. 10 - RH removal of the WGs vertical extensions

In Fig. 11 is depicted the operations used by the RH arm to remove the WGs horizontal extensions. After the removable of all WGs extensions the neutron shielding can be accessed and removed.
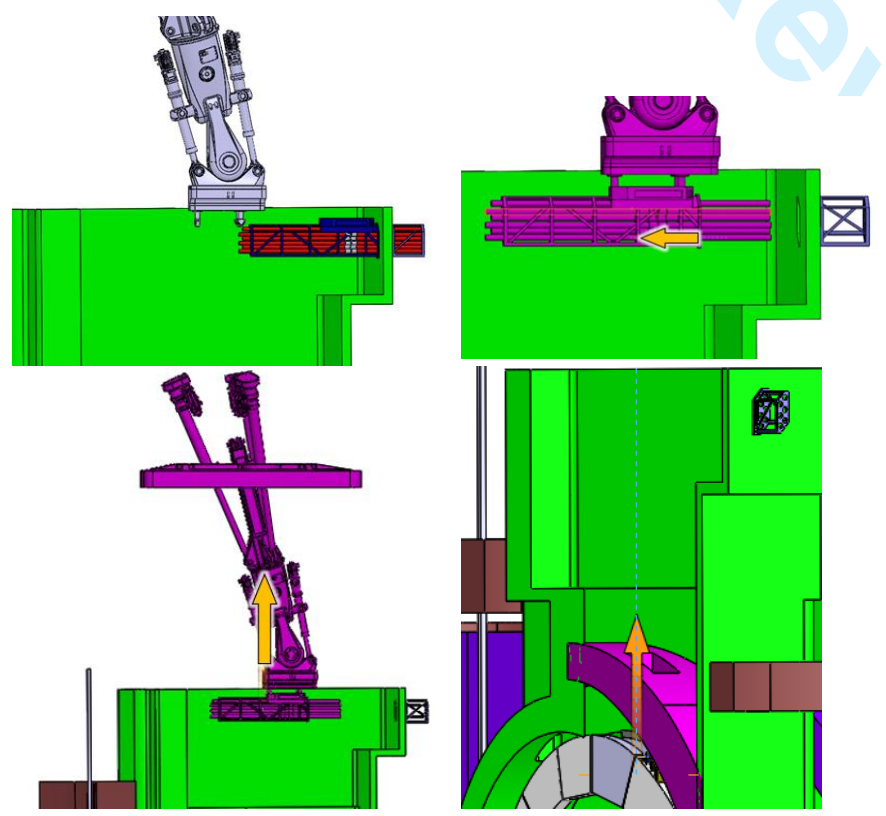

Fig. 11 - Illustration of the remote handling removal of the WGs horizontal extensions. After the WGs are removed the port is clear for schedule RH services e.g., BB replacement.

The access to the port side in order to fix and align the last segments of WGs has not yet been investigated. It is also anticipated that some of the RH solutions for installation and repairing the $\mathrm{BB}$ services pipes exiting from the port side could be used for the remote access to the port side flange.

\section{NEUTRON HEATING AND COOLING CONCEPT}

Neutron and induced gamma fluxes were estimated using a standard Monte Carlo code (MCNP6). The simplified geometric input was prepared adapting from the CATIA to the MCNP geometry handler. A standard DEMO equilibria was used for the neutron source and a material composition based on the HCLL blanket. The antennas and waveguides material is EUROFER (90\%) and He (10\%, @ 80 bar) is considered as coolant. The SC is installed between two HCLL BB sectors. The calculated neutron and gamma fluxes are presented in Fig. 12.
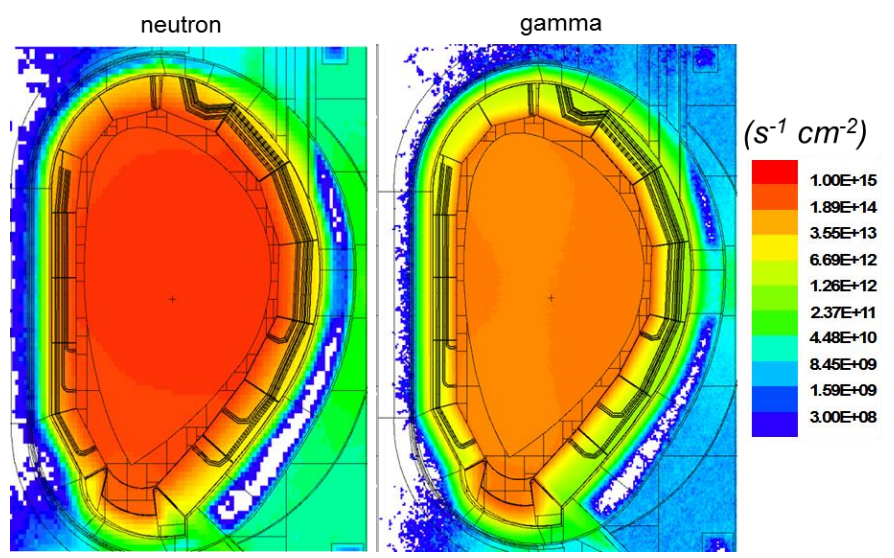

Fig. 12 - Neutron and gamma fluxes calculated with the SC. The segmentation used was of 14 BB modules (later updated to the 2017 model)

The results show that there is no significant increase of the radiation in the vessel due to the presence of the waveguides (empty volumes). The induced nuclear heating on the antennas and waveguides at different poloidal locations was calculated. The results presented in Fig. 13 indicate that the average heat loads in the antennas is similar to the volumetric heat loads in the $\mathrm{BB}$ at an equivalent location. The same holds for the waveguides nuclear heating, similar to the local BB. These results indicate the possibility to develop a BB-like cooling design for the SC.
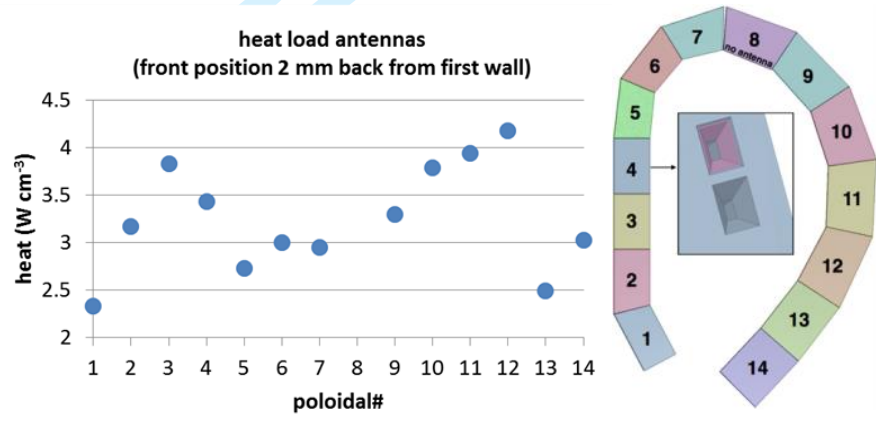

Fig. 13 - Average heat loads in the antennas as a function of the poloidal location.

The cooling concept proposed is based on the same boundary conditions as for the BB modules. Since the SC is attached to a common BSS the goal is to not raise thermal stresses neither different mechanical dynamics during operation, from thermal cycling, and RH manipulation. Another requirement is that the density of the SC must be similar to the BB average density. In addition, the He coolant 
will have to be contained in a double barrier arrangement. Last but not least, the SC has to perform an equivalent shielding function as the BB equivalent (removed for inserting the SC). The design that is being considered for the core of the SC is presented in Fig. 14. The vertical EUROFER shielding plates

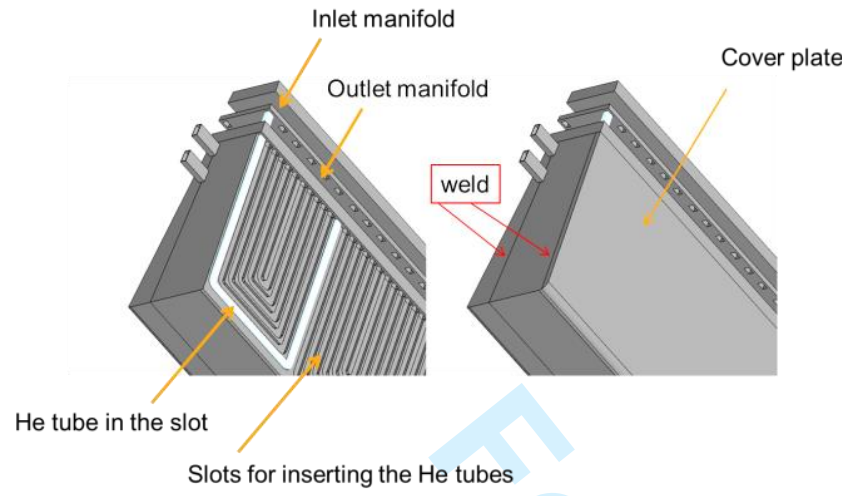

Fig. 14 - Double containment barrier cooling system for the SC modules. The shielding plates contain groves for receiving the He pipes. A cover plate is welded to hermetically close the coolant pipes establishing the second containment barrier.

have a mask of groves where the He pipes are incrusted. A cover plate seals the volume establishing a second confinement barrier. The present design assumes an independent first wall for the SC modules. The cooling approach for that component follows the same approach as for the BB first wall and the pipe dimensions are kept the same as for the first wall. In Fig. 15 is depicted the structure of the SC module with the first wall.

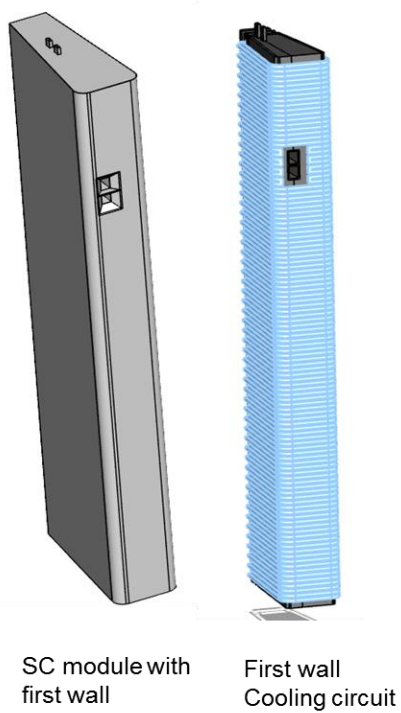

Fig. 15 - SC module with first wall (left) and FW cooling channels (right).

In order to evaluate the performance of the cooling design proposed an updated MCNP6 calculation was done and the heat deposition calculated for the detailed model of the SC module (Fig. 16). The results presented are preliminar corresponding to the output of the proposed design without performing any optimization.
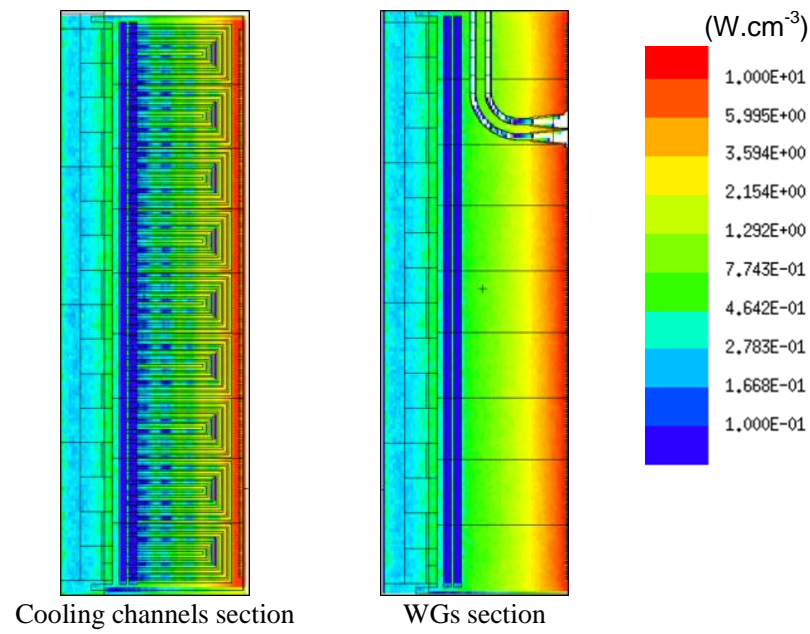

Fig. 16 - Nuclear heat load in the SC module using a new poloidal segmentation model with $17 \mathrm{BB}$ modules (plasma on the right side).

In the above calculation the antennas heat load volume was radially divided in four regions in order to more easily detect any hot spots. In Fig. 17 are depicted the final temperatures on the SC elements obtained for the boundary conditions assumed (similar to the BB modules with rectangular cooling pipes $\left(8 \times 13 \mathrm{~mm}^{2}\right)$, average temperature $400{ }^{\circ} \mathrm{C}, \mathrm{He} @ 80 \mathrm{bar}$, inlet $300{ }^{\circ} \mathrm{C}$; outlet $500{ }^{\circ} \mathrm{C}$, plasma radiation $500 \mathrm{~kW} . \mathrm{m}^{-2}$ ). The results show that the average operating temperature at the first wall is circa $450{ }^{\circ} \mathrm{C}$ increasing towards the plasma. However, for the $\mathrm{FW}$ face in front of the plasma the temperature is clearly higher than $550{ }^{\circ} \mathrm{C}$. In order to improve the cooling the first approach to be investigated will be to increase the $\mathrm{He}$ velocity (increase of heat transfer coeficient) or increase the volume ratio (He/Eurofer) by re-designing the cooling channels. The hot spot areas on the top and bottom of the first wall indicate that an improved cooling design needs to be routed in that area. As for the inner body of the SC module and WG's the temperature is in the range of $400{ }^{\circ} \mathrm{C}$, except for the localized hot spots in the antennas calling for active colling and/or a closer proximity with the cooling channels.
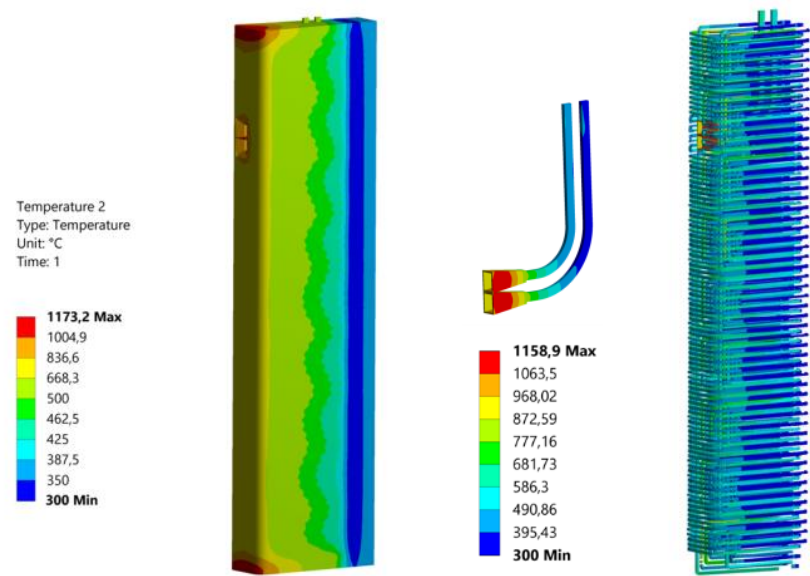

Fig. 17 - Heat loads in the SC module first wall (left), antennas and SC components (right). 


\section{CONCLUSIONS}

Reflectometry is foreseen as one major diagnostic for DEMO. It shall provide measurements of the plasma density in the pedestal region, and the plasma position and shape. A preliminary study show that the range of spatial locations for operating reflectometry with sufficient performance is mostly located at equatorial level and at the mid region between upper and equatorial port. A concept for integration of the waveguides and antennas has been developed using a Slim Cassette as an independent component. The attachment and cooling of the SC are shared with the BB modules via a common back support structure. The remote handling compatibility was investigated and the whole system removal/installation was possible with the existing tools. There are particular locations such the side of the port where a dedicated WGs flange will be placed for which the RH procedure and vacuum interface still needs to be developed in more detail. As for the IB WGs extensions several interfaces with BB pipe services are still to be addressed. The MCNP simulations were performed and the results on neutron and gamma fluxes were used to estimate the heat load in the diagnostic components and SC module. The cooling concept for the SC based on a double containment barrier was proposed and analyzed with ANSYS software. The preliminary results indicate that the average temperature of the $\mathrm{SC}$ module first wall (worst case scenario) is higher by $50{ }^{\circ} \mathrm{C}$ than the required value of $400{ }^{\circ} \mathrm{C}$. A $50 \%$ increase on He flux brought the average SC FW temperature within the operation requirements. The final average temperature and the hotpots in the antennas and FW may be mitigated in a second iteration by some adjustments on the cooling pipes dimensions and routing and using $\mathrm{W}$ for the antenna material.

\section{ACKNOWLEDGMENT}

"This work has been carried out within the framework of the EUROfusion Consortium and has received funding from the Euratom research and training programme 2014-2018 under grant agreement No 633053. The views and opinions expressed herein do not necessarily reflect those of the European Commission."

\section{REFERENCES}

[1] F.P. Orsitto et al., "Diagnostics and control for the steady state and pulsed tokamak DEMO", Nucl. Fusion 56 (2016) 026009 (23pp), doi:10.1088/0029-5515/56/2/026009

[2] J. Santos et al., "Reflectometry-based plasma position feedback control demonstration at ASDEX Upgrade", Nucl. Fusion 52 (2012) 032003 (6pp), doi:10.1088/0029-5515/52/3/032003.

[3] G. Vayakis et al., "Reflectometry on ITER", ITER Joint Central Team and Home Teams Review of Scientific Instruments 68, 435 (1997); doi: 10.1063/1.1148220

[4] J. Keep et al., "Remote handling of DEMO breeder blanket segments: Blanket transporter conceptual studies", Fusion Engineering and Design, Available online 12 February 2017, ISSN 0920-3796, https://doi.org/10.1016/j.fusengdes.2017.02.016. 